\title{
GERMINAÇÃO DE SEMENTES E QUALIDADE DE MUDAS DE Plinia trunciflora (JABUTICABEIRA) EM FUNÇÃO DE DIFERENTES TRATAMENTOS PRÉ-GERMINATIVOS
}

\author{
Überson Boaretto Rossa ${ }^{1}$, Gilmar Paulinho Triches ${ }^{1}$, Fernando Grossi ${ }^{2}$, Antonio Carlos Nogueira ${ }^{2}$, \\ Carlos Bruno Reissmann ${ }^{3}$, Michele Ribeiro Ramos ${ }^{4}$ \\ ${ }^{1}$ Lic. em Ciências Agrárias, M.Sc., Instituto Federal de Educação Ciência e Tecnologia, IFET, Rio do Sul, SC, Brasil - \\ boarettorossa@gmail.com; gilmartriches@ hotmail.com \\ ${ }^{2}$ Eng. Florestal, Dr., Depto. de Ciências Florestais, UFPR, Curitiba, PR, Brasil - f_grossi@ufpr.br - nogueira@ufpr.br \\ ${ }^{3}$ Eng. Florestal, Dr., Depto. de Solos, UFPR, Curitiba, PR, Brasil - reissmann @ufpr.br \\ ${ }^{4}$ Eng ${ }^{a}$ Agrônoma, M.Sc., Doutoranda em Eng. Florestal, UFPR - miagro2@ yahoo.com.br \\ Recebido para publicação: 05/11/2008 - Aceito para publicação: 30/09/2009
}

\begin{abstract}
A formação de mudas florestais de boa qualidade morfofisiológica envolve os processos de germinação de sementes, que por sua vez é dependente de potencialidades genéticas. Algumas ações no tratamento prévio de sementes podem auxiliar na germinação. Este trabalho objetivou avaliar a germinação de sementes e qualidade inicial de mudas de jabuticabeira sob diferentes tratamentos prégerminativos: T1 - sementes completamente limpas; T2 - sementes oxidadas com o fruto macerado; T3 - sementes com polpa; e T4 - semeadura do fruto com semente. O experimento foi conduzido durante o período de outubro de 2007 a agosto de 2008. O delineamento experimental foi inteiramente casualizado, com quatro repetições. Realizou-se a avaliação da percentagem de germinação aos 40, 60 e 90 dias da semeadura, e o cálculo do índice de velocidade de emergência realizado ao final do período. Aos 306 dias após a semeadura foram avaliados os parâmetros morfológicos das mudas, suas relações e o índice de qualidade de Dickson para mudas. Comprovouse que as sementes completamente limpas (T1) ou com endocarpo aderido (T3) foram os tratamentos prévios mais eficazes para os processos de germinação e qualidade da muda formada.

Palavras-chave: Jabuticaba; frutífera nativa; propagação sexuada; produção de mudas.
\end{abstract}

\begin{abstract}
Germination of seeds and seedling quality of Plinia trunciflora under different pre-germinative treatments. The formation of forest seedlings of good morphophysiological quality involves processes of seed germination, which in turn is dependent on genetic potential. Some actions in the pretreatment of seed germination can help. This study aimed to evaluate the germination and initial quality of seedlings under different treatments jabuticaba pre-germinated: T1 - seeds thoroughly cleaned; T2 seeds oxidized with the fruit macerate; T3 - pulp with seeds, and T4 - the fruit with seed sowing. The experiment was conducted during the period from October 2007 to August 2008. The experimental design was a completely randomized with four treatments in 4 replicates. It was calculated of the percentage of germination at 40,60 and 90 days, and the analysis of the index emergency speed at the end of the period. At 306 days after sowing they were assessed the morphological parameters of seedlings, their relationships and quality index of Dickson for seedlings. It has been proved that the seeds thoroughly cleaned and cored joined with the previous treatments were the most effective in the process of germination and seedling quality.

Keywords: Jabuticaba fruits; native fruit; sexual propagation; production of seedlings.
\end{abstract}

\section{INTRODUÇÃO}

A flora brasileira é rica em frutas silvestres comestíveis, as quais constituem um patrimônio genético e cultural de inestimável valor (MIELKE et al., 1990). Dentre as espécies nativas de importância regional no sul do Brasil, destaca-se a jabuticabeira (Plinia sp.), pertencente à família Myrtaceae (MATTOS, 1978). Encontrada desde o estado do Pará até o Rio Grande do Sul, Paraguai e Argentina, 
trata-se de fruteira que apresenta porte médio a grande, com 6 a $8 \mathrm{~m}$ de altura, podendo alcançar até $12 \mathrm{~m}$, tendo como tendência típica o hábito de frutificação nos ramos com ruptura da casca (LEGRAND; KLEIN, 1978; ROTMAN, 1982; LORENZI et al., 2006).

A família Myrtaceae possui cerca de 140 gêneros e mais de 3000 espécies, e seus dois principais centros de dispersão são América e Austrália (JOLY, 1993; RIBEIRO, 1999). Várias espécies dessa família, principalmente as nativas do Brasil, têm frutos comestíveis, tais como goiabeira, araçazeiro, cabeludinha, guabirobeira, cambucizeiro e jabuticabeira, entre outras (JOLY, 1993). Os frutos produzidos pelas plantas da família das Myrtaceae apresentam potencial de comercialização em função de suas características organolépticas, principalmente em nichos de mercado ávidos por novidades e em épocas do ano em que não há outras frutas no mercado (MAGALHÃES et al., 1996).

A jabuticabeira é conhecida há quase cinco séculos. Seu nome é originário do tupi, "iapoti'kaba", que significa "fruta em botão" (MENDONÇA, 2000). Essa família é uma das mais utilizadas pelos índios, provavelmente devido à facilidade com que suas espécies aparecem espontaneamente nos roçados e capoeiras (ANJOS, 1998).

Segundo Mattos (1978), são conhecidas em torno de 9 espécies de jabuticabeira, entre elas Plinia trunciflora (DC.) Berg (jabuticaba-de-cabinho), Plinia cauliflora (jabuticaba-paulista ou jabuticaba-açu) e Plinia jaboticaba (Vell.) (jabuticaba-sabará). Todas produzem frutos apropriados tanto para consumo in natura como para a indústria de fabricação de geleias, vinhos e licores caseiros (MATTOS, 1983; DONADIO, 1983).

A alteração nomenclatural do gênero Myrciaria (BERG, 1857) para o gênero Plinia foi proposta por Sobral (1985).

Um dos problemas enfrentados para a expansão dos pomares comerciais é a obtenção de mudas. A maioria dos viveiristas opta pela propagação através de sementes, que conservam as características genéticas da planta matriz (ANDERSEN; ANDERSEN, 1988; ANTUNES et al., 1995), porém essa espécie apresenta baixa densidade de ocorrência de matrizes produtoras de sementes, dificultando a obtenção de sementes em quantidade que permita a produção de mudas em larga escala, seja para aproveitamento comercial, com o plantio de pomares de produção de frutas, seja para aproveitamento em programas de repovoamento vegetal.

Essa fruteira também pode ser multiplicada assexualmente por enxertia, mergulhia e estaquia (GOMES, 1987; SCARPARE FILHO et al., 1999; DANNER et al., 2006). Porém, em virtude da baixa importância econômica e por ser considerada espécie de difícil enraizamento, o método amplamente empregado para produzir mudas de jabuticabeira é aquele em que se utilizam sementes, obtendo-se mudas denominadas "pés-franco" (MANICA, 2000).

Muitos estudos têm sido realizados com o intuito de reduzir o tempo necessário entre a semeadura e a emergência das plântulas, bem como para aumentar a tolerância das sementes às condições adversas durante a germinação. Alguns tratamentos têm se mostrado eficientes nesse sentido, apresentando resultados bastante promissores com sementes de diversas espécies (KHAN, 1992).

Para a jabuticabeira não existem muitos estudos sobre o melhor método de semeadura, ou ainda de técnicas pré-germinativas que melhor induzam o aumento do potencial de desempenho das sementes. Aparentemente, as diferentes espécies de jabuticabeira não apresentam dormência das sementes, porém estudos podem indicar técnicas para melhorar a sincronização de germinação das sementes e o vigor das mudas formadas, aumentando o seu potencial de desempenho e, por conseguinte, a uniformidade das plantas em condições de campo.

A muda é o insumo mais importante na implantação de um pomar. Mudas produzidas com qualidade e adequadamente manejadas originam pomares produtivos e rentáveis (PASQUAL et al., 2001; CHALFUN; PIO, 2002). Duryea (1984) e Mexal; Landis (1990) também sugerem que a qualidade das mudas é de fundamental importância, pois estão ligadas ao sucesso do reflorestamento.

Entre os fatores que influenciam na produção de mudas de espécies florestais, destacam-se, além das sementes, os recipientes e o substrato utilizado, os quais vão refletir diretamente na qualidade do produto final (CARNEIRO, 1995).

Para Rose et al. (1990), uma muda padrão é determinada por características morfológicas (estruturais) e fisiológicas. Estas, por sua vez, são definidas por fatores genéticos (propágulos) e ambientais (tratos culturais no viveiro). 
Os principais parâmetros que determinam a qualidade das mudas são a altura, o diâmetro do coleto, o peso da parte aérea e das raízes e as correlações entre esses parâmetros (CARNEIRO, 1995).

Poucos são os trabalhos que associam os tratamentos pré-germinativos à germinação da semente e qualidade de mudas de jabuticabeiras, faltando informações básicas sobre esdas relações.

Sendo assim, o presente trabalho teve como objetivo avaliar a germinação e a qualidade inicial de mudas de jabuticabeira sob diferentes tratamentos pré-germinativos.

\section{METODOLOGIA}

O experimento foi realizado durante o período de outubro de 2007 a agosto de 2008 , sendo conduzido em viveiro de produção de mudas localizado em $27^{\circ} 11^{\prime} \mathrm{S}$ e $49^{\circ} 39^{\prime} \mathrm{W}$, numa altitude de $701 \mathrm{~m}$, instalado no Instituto Federal de Educação, Ciência e Tecnologia, Campus de Rio do Sul, no município de Rio do Sul, SC.

Frutos maduros de jabuticaba foram coletados em uma única árvore plantada em pomar no município de Rio do Sul. Foram selecionados os frutos de maior tamanho e ausência de danos visíveis. As sementes foram semeadas sob distintos tratamentos: T1: sementes completamente limpas; T2: sementes oxidadas com o fruto macerado; T3: sementes com polpa (endocarpo aderido); e T4: semeadura do fruto com semente.

Para T1, as sementes foram extraídas dos frutos e limpas completamente, utilizando-se uma flanela para a retirada do endocarpo aderido ao tegumento da semente. Para T2, os frutos foram macerados juntamente com o bagaço (epicarpo, mesocarpo e endocarpo) e permaneceram em balde plástico durante 7 dias, para promoção da oxidação. Após esse período, as sementes foram retiradas da fermentação e lavadas sob peneira com água corrente e logo em seguida semeadas. As sementes do T3 foram semeadas com o endocarpo aderido logo após a retirada da casca (epicarpo).

A semeadura foi realizada em 20 de outubro de 2007, utilizando-se tubetes de polipropileno de $180 \mathrm{~cm}^{3}$, tendo como substrato uma mistura de Substrato Florestal Plantmax (50\% v/v), composto orgânico peneirado $(30 \% \mathrm{v} / \mathrm{v})$ e vermiculita de granulometria média $(20 \% \mathrm{v} / \mathrm{v})$. As características químicas e físicas do substrato estão apresentadas na tabela 1.

Os vasos foram preenchidos e logo submetidos à mesa compactadora por 10 segundos, objetivando densidade uniforme de substrato. Após a semeadura, as bandejas com os vasos foram mantidas sob sombreamento de $50 \%$, e a umidade do substrato foi mantida por sistema de irrigação via microaspersão.

O delineamento experimental foi o inteiramente casualizado, com quatro repetições, e a unidade experimental foi constituída pelo conjunto de 25 tubetes.

Procedeu-se o calculo da percentagem de germinação (PG) aos 40,60 e 90 dias após a semeadura, e do índice de velocidade de emergência (IVE) realizado ao final do período. O IVE foi calculado segundo Popinigis (1977), sendo determinado pela divisão do número de plântulas emergidas pelo número de dias transcorridos da data de semeadura, em cada data de avaliação. O total desses valores somados foi considerado o índice de velocidade de emergência. A contagem de folhas também foi realizada ao final do experimento, considerada como indicativo de biomassa produzida.

Decorridos 306 dias da semeadura (em 20/08/2008), foram analisadas as variáveis altura total (H), diâmetro do coleto (DC), relação entre altura e diâmetro do coleto (H/DC), matéria fresca da parte aérea (MFPA), matéria seca da parte aérea (MSPA), matéria seca da raiz (MSR), matéria seca total (MST), calculando-se o índice de qualidade de mudas segundo Dickson (1960). Coletaram-se dados de altura da parte aérea das mudas, medindo-se as mesmas com régua $(\mathrm{cm})$, do nível do solo até o ápice, e o diâmetro do coleto foi obtido com paquímetro $(\mathrm{mm})$ a $0,5 \mathrm{~cm}$ do solo. Em seguida, determinou-se a matéria fresca da parte aérea, com balança de precisão com três casas decimais. As raízes foram destorroadas e lavadas, utilizando-se peneiras de $2 \mathrm{~mm}$ para evitar possíveis perdas de radículas. As amostras da parte aérea e de raízes foram acondicionadas em sacos de papel pardo e secas em estufa a $60{ }^{\circ} \mathrm{C}$, com ventilação forçada até peso constante, que ocorreu ao final do terceiro dia de secagem.

Os dados foram submetidos a análise de variância e as médias comparadas pelo teste de Tukey a $5 \%$. 
Tabela 1. Análise química e física de matérias-primas utilizadas no substrato nos experimentos de germinação de sementes de jabuticabeira (Plinia trunciflora) em função de diferentes tratamentos pré-germinativos.

Table 1. Chemical and physical analysis of the components utilized in the experimental substrate for the jaboticaba (Plinia trunciflora) seeds production according to different treatments pregerminated.

\begin{tabular}{|c|c|c|c|c|c|c|c|c|c|c|c|c|c|}
\hline \multirow{2}{*}{ Substrato } & \multirow{2}{*}{$\begin{array}{c}\mathrm{pH} \\
\mathrm{H}_{2} \mathrm{O}\end{array}$} & \multirow{2}{*}{$\begin{array}{l}\text { Índice } \\
\text { SMP }\end{array}$} & $\mathrm{Ca}$ & $\mathrm{Mg}$ & $\mathbf{A l}$ & $\mathrm{H}+\mathrm{Al}$ & CTC & \multicolumn{2}{|c|}{ Saturação (\%) } & M.O. & Argila & $\mathbf{P}$ & $\mathbf{K}$ \\
\hline & & & & \multicolumn{4}{|c|}{ 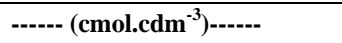 } & $\overline{A l}$ & Base & \multicolumn{2}{|c|}{---- (\%) - } & \multicolumn{2}{|c|}{--- $\left(\right.$ mg.dm $\left.^{-3}\right)---$} \\
\hline $\begin{array}{l}\text { Composto } \\
\text { Orgânico }\end{array}$ & 6,3 & 6,8 & 7,4 & 4,4 & 0,0 & 1,7 & 16,5 & 0,0 & 89,5 & 7,8 & 27 & 560 & 1160 \\
\hline $\begin{array}{l}\text { Florestal } \\
\text { Plantmax }\end{array}$ & 4,0 & 4,2 & 7,6 & 4,4 & 1,7 & 34,4 & 47,6 & 11,5 & 27,6 & 10,2 & 7 & 172 & 440 \\
\hline Vermiculita & 6,9 & 7,6 & 6,2 & 3,6 & 0,0 & 0,7 & 10,6 & 0,0 & 93,4 & 0,1 & 1 & 2 & 28 \\
\hline
\end{tabular}

\section{RESULTADOS E DISCUSSÃO}

\section{Fase I (germinação)}

Os resultados de porcentagem de germinação e índice de velocidade de emergência, após os tratamentos pré-germinativos, estão ilustrados na tabela 2.

Tabela 2. Percentagens de germinação (PG) aos 40, 60 e 90 dias após semeadura, índice de velocidade de emergência (IVE) e número de folhas (NF) de mudas de Plinia trunciflora (jabuticabeira) em função de diferentes tratamentos pré-germinativos.

Table 2. Germination percentage at 40,60 and 90 days, speed emergency index and number of leaves, seedlings of Plinia trunciflora (jabuticabeira) according to treatments pre-germinated.

\begin{tabular}{|c|c|c|c|c|c|}
\hline \multirow{2}{*}{ Tratamento } & PG 40 & PG 60 & PG 90 & \multirow{2}{*}{ IVE } & \multirow{2}{*}{ NF } \\
\hline & \multicolumn{3}{|c|}{------- \% ------- } & & \\
\hline T1 - Sementes completamente limpas & $76 \mathrm{a}$ & $89 \mathrm{a}$ & $98 \mathrm{a}$ & $1,12 \mathrm{a}$ & $38 \mathrm{a}$ \\
\hline $\mathrm{T} 2$ - Sementes oxidadas com o fruto macerado & $2 \mathrm{~b}$ & $55 \mathrm{~b}$ & $61 \mathrm{~b}$ & $0,41 \mathrm{~b}$ & $28 \mathrm{~b}$ \\
\hline T3 - Sementes com endocarpo aderido & $68 \mathrm{a}$ & $77 \mathrm{a}$ & $85 \mathrm{a}$ & $0,98 \mathrm{a}$ & $39 \mathrm{a}$ \\
\hline T4 - Semeadura do fruto com semente & $0 \mathrm{~b}$ & $44 \mathrm{~b}$ & $52 \mathrm{~b}$ & $0,33 \mathrm{~b}$ & $30 \mathrm{~b}$ \\
\hline $\mathrm{CV}(\%)$ & 21,69 & 15,42 & 12,91 & 13,44 & 9,33 \\
\hline
\end{tabular}

Médias seguidas pela mesma letra não diferem entre si pelo teste de Tukey a 5\% de probabilidade.

As sementes completamente limpas (T1) apresentaram a maior percentagem de germinação (PG) em todos os períodos analisados após a semeadura: 76, 89 e $98 \%$ aos 40, 60 e 90 dias, respectivamente. Não houve diferença significativa entre as sementes completamente limpas (T1) quando comparadas nesse mesmo período com as sementes com o endocarpo aderido (T3), que apresentaram PG de 68, 77 e $85 \%$ para o período de 40, 60 e 90 dias, respectivamente. Para o índice de velocidade de germinação (IVG) também não houve diferenças entre os tratamentos T1 e T3. Alexandre et al. (2006), ao analisarem a semeadura, em diferentes estádios de maturação do fruto de jabuticabeira em dois tipos de substrato, obtiveram como resultado PG de 66,43\% e 41,59\% aos 36 dias após a semeadura, e IVG de 2,17 e 0,94, utilizando como substrato vermiculita e areia, respectivamente. Os valores de PG e IVG relativos à semeadura feita em vermiculita, naquele estudo, são equivalentes quando os comparamos com os levantados neste trabalho, possivelmente pelo substrato ter características semelhantes de porosidade e capacidade de retenção de umidade, que, segundo Kämpf (2000), são características que favorecem a germinação e o crescimento inicial das plântulas.

Também Andrade (2003), ao testar a influência da temperatura na germinação de sementes de jabuticabeira, observou um PG de 76,62\% em temperatura de $15{ }^{\circ} \mathrm{C}$, em substrato de vermiculita, utilizando sementes completamente limpas, corroborando os resultados obtidos neste trabalho para o T1 aos 40 dias após semeadura, que foi de $76 \%$.

Quanto à variável número de folhas (NF), não houve diferenças entre as mudas geradas, com média de 38 e 39 folhas por plantas, respectivamente, para as sementes completamente limpas (T1) e sementes com endocarpo aderido (T3). O incremento da produção de biomassa da parte aérea, indicado 
pelo NF, pode ser devido à precocidade de germinação das sementes desses tratamentos, associada a um maior desenvolvimento das raízes e consequentemente maior crescimento da parte aérea.

Para as sementes oxidadas com o fruto macerado (T2) e para as sementes que foram semeadas juntamente com os frutos (T4), houve baixo percentual de germinação aos 40 dias, demonstrando, assim, que o tegumento, possivelmente, dificulta a embebição de água pela semente, retardando a germinação. As percentagens corresponderam a 2 e $0 \%$ para T2 e T4, respectivamente, nesse período. Ainda para T2 e T3, avaliados aos 60 dias, o PG correspondeu a 55 e 44\%, respectivamente. Esses índices de PG aumentaram aos 90 dias, ficando em 61 e 52\% para T2 e T4, respectivamente. Não houve diferença estatística para as percentagens de PG em nenhum dos períodos avaliados, bem como para IVG e NF, entre os tratamentos T2 e T4.

Observou-se que as sementes oxidadas com o fruto macerado (T2), ocasionalmente não germinadas aos 40 dias, não são necessariamente inviáveis: o que ocorre é um retardamento do processo de germinação, pois elas, aos 90 dias, apresentaram PG considerável. Comportamento semelhante foi verificado para as sementes semeadas juntamente com o fruto (T4).

A permeabilidade do tegumento pode ser o fator preponderante para a germinação da semente, cuja embebição é a primeira etapa nesse processo (CARVALHO; NAKAGAWA, 1980), quando comparadas as sementes com o endocarpo aderido (T3) e as sementes semeadas com o fruto (T4).

As sementes de jabuticabeira submetidas a diferentes tratamentos pré-germinativos apresentaram níveis aceitáveis de germinação, não sendo constatado nenhum nível de dormência tegumentar. Observase que as sementes completamente limpas e as sementes com endocarpo aderido foram os tratamentos prévios mais eficazes para os processos de germinação (PG e IVG).

\section{Fase II (qualidade de mudas)}

De um modo geral, houve efeito de T1 e T3 (sementes completamente limpas e sementes com endocarpo aderidas) sobre todos os parâmetros biométricos que indicam o desenvolvimento das mudas de jabuticabeira (Tabela 3).

Tabela 3. Médias das variáveis altura total $(\mathrm{H})$, diâmetro do coleto $(\mathrm{DC})$, matéria fresca da parte aérea (MFPA), matéria seca da parte aérea (MSPA), matéria seca da raiz (MSR), matéria seca total (MST) das mudas de jabuticaba provenientes de diferentes tratamentos pré-germinativos de semente.

Table 3. Means of the variables total height, collar diameter, fresh biomass of the above ground part, dry biomass of the above ground part, shoot dry matter, total dry matter seedlings of jaboticaba from different pre-treatment for seed germination.

\begin{tabular}{|c|c|c|c|c|c|c|c|c|}
\hline \multirow{2}{*}{ Tratamento } & \multicolumn{6}{|c|}{ Parâmetros biométricos } & \multicolumn{2}{|c|}{$\begin{array}{l}\text { Îndices de } \\
\text { qualidade }\end{array}$} \\
\hline & $\begin{array}{c}\mathbf{H} \\
--\mathbf{c m}--\end{array}$ & $\begin{array}{c}\text { DC } \\
-\mathbf{m m}-\end{array}$ & MFPA & MSPA & MSR & MST & H/DC & IQD \\
\hline $\begin{array}{l}\text { T1 - Sementes } \\
\text { completamente } \\
\text { limpas }\end{array}$ & $22,25 \mathrm{a}$ & $3,125 \mathrm{a}$ & $7,214 \mathrm{a}$ & $2,404 \mathrm{a}$ & $0,713 \mathrm{a}$ & $3,117 \mathrm{a}$ & $7,19 \mathrm{a}$ & $0,30 \mathrm{a}$ \\
\hline $\begin{array}{l}\mathrm{T} 2-\text { Sementes } \\
\text { oxidadas com o fruto } \\
\text { macerado }\end{array}$ & $15,25 b c$ & $2,575 \mathrm{~b}$ & $4,314 \mathrm{~b}$ & $1,348 \mathrm{~b}$ & $0,336 \mathrm{~b}$ & $1,684 \mathrm{~b}$ & $5,92 \mathrm{ab}$ & $0,17 \mathrm{~b}$ \\
\hline $\begin{array}{l}\text { T3 - Sementes com } \\
\text { endocarpo aderido }\end{array}$ & $20,25 \mathrm{ab}$ & $2,850 \mathrm{ab}$ & $6,922 \mathrm{a}$ & $2,128 \mathrm{a}$ & $0,669 \mathrm{a}$ & $2,798 \mathrm{a}$ & $7,07 \mathrm{a}$ & $0,27 \mathrm{a}$ \\
\hline $\begin{array}{l}\mathrm{T} 4 \text { - Semeadura do } \\
\text { fruto com semente }\end{array}$ & $12,25 \mathrm{c}$ & $2,375 \mathrm{~b}$ & $4,572 \mathrm{~b}$ & $1,475 \mathrm{~b}$ & $0,305 \mathrm{~b}$ & $1,781 \mathrm{~b}$ & $5,11 \mathrm{~b}$ & $0,18 \mathrm{~b}$ \\
\hline CV (\%) & 15,91 & 9,04 & 10,76 & 10,72 & 16,23 & 7,190 & 14,78 & 18,97 \\
\hline
\end{tabular}

Médias seguidas pela mesma letra não diferem entre si pelo teste de Tukey a $5 \%$ de probabilidade.

No que se refere à altura das mudas produzidas em $\mathrm{T} 1$ e $\mathrm{T} 3$, elas foram estatisticamente superiores a T2 e T4. Segundo MEXAL; ANDS (1990), a altura da parte aérea das mudas fornece uma excelente estimativa da predição do crescimento inicial no campo, sendo tecnicamente aceita como uma boa medida do potencial de desempenho das mudas. Contudo, recomenda-se que os valores devem ser analisados combinados com o diâmetro do coleto (CARNEIRO, 1995). Danner et al. (2007), estudando a 
formação de mudas de jabuticabeira em diferentes substratos, após 12 meses de semeadura, obtiveram respostas semelhantes para os parâmetros de altura da muda e diâmetro do caule.

Os resultados obtidos quanto à avaliação do diâmetro do coleto das mudas de jaboticaba, evidenciou que as mudas produzidas a partir do T1 foram superiores aos tratamentos T2 e T4. Quando relacionada a altura das mudas com o diâmetro do coleto, apenas o T4 diferiu dos demais. O diâmetro do coleto é facilmente mensurável, considerado um dos mais importantes parâmetros para estimar o desenvolvimento da muda de diferentes espécies florestais (GOMES et al., 2002).

Nesse sentido, este trabalho sugere que, para a produção de mudas de jabuticabeira com bom desenvolvimento em altura e diâmetro do coleto, não se deve proceder semeadura do fruto sem qualquer pré-tratamento para retirada do epicarpo e endocarpo.

O valor resultante da divisão da altura da parte aérea pelo diâmetro do colo exprime um equilíbrio de crescimento, relacionando esses dois importantes parâmetros morfológicos em apenas um índice, sendo que um bom resultado para a relação altura da parte aérea e diâmetro do colo pode ser obtido quando os valores se encontram entre 5,4 e 8,1 (CARNEIRO, 1995). Essa relação, também denominada de quociente de robustez, é considerada uma dos mais preciosas, pois fornece informações de quanto delgada está a muda (JOHNSON; CLINE, 1991).

Na tabela 3, consta que neste estudo, em todos os tratamentos, o índice H/DC foi menor que 10, padrão recomendado por Birchler et al. (1998), ocorrendo um equilíbrio entre os tratamentos, com as mudas geradas pela semeadura de sementes oxidadas não diferindo estatisticamente das demais. Apenas as mudas da semeadura do fruto com semente mostraram um equilíbrio inferior aos demais tipos de semeadura.

As sementes completamente limpas e com endocarpo aderido fizeram com que as mudas tivessem uma maior produção de biomassa quando analisadas todas as variáveis de peso. Provavelmente, a diferença no ganho de biomassa ocorreu devido ao processo de germinação iniciar antes das demais (PG de $76 \%$ e $68 \%$ aos 40 dias para o $\mathrm{T} 1$ e T3, respectivamente), tendo as plantas maior tempo de conversão de biomassa, se comparado com as outras que germinaram posteriormente.

$\mathrm{Na}$ determinação da qualidade das mudas, os parâmetros utilizados baseiam-se em aspectos morfológicos (GOMES et al., 2002), os quais dependem também da carga genética e da procedência das sementes, das condições ambientais e das técnicas de produção em nível de viveiro, sendo que muitas vezes essas características morfológicas são as mais adequadas para avaliar o desempenho das mudas após o plantio no campo (PARVIAINEN, 1981).

As mudas produzidas a partir de semeadura do fruto não tiveram um bom desenvolvimento. Isso pode ter sido em função do retardo da germinação das sementes, provavelmente pela impermeabilização das partes do fruto que revestem a semente. No entanto, ao avaliar as percentagens de germinação desse tratamento, verifica-se que houve um bom índice (52\%) aos 90 dias após a semeadura.

Para o índice de qualidade de Dickson, houve discriminação para as mudas de T1 e T3, sendo registrado maior índice para as mudas produzidas a partir da semeadura de sementes completamente limpas $(0,30)$ e semeadura de sementes com endocarpo aderido $(0,27)$, sem diferença estatística entre elas. Portanto, pode ser dispensada a retirada da polpa para a semeadura, sem prejuízo ao período de germinação da semente, tendo em vista a economia de mão de obra para essa atividade no viveiro.

Esses resultados mostram que as diferenças morfológicas iniciais das mudas, medidas pelos parâmetros biométricos, bem como pelos índices de qualidade de mudas, são afetadas pelos distintos tratamentos pré-germinativos da semente.

\section{CONCLUSÃo}

- Os resultados do presente trabalho demonstram que a semeadura de sementes totalmente limpas ou com polpa aderida permite a formação de mudas de jabuticabeira de boa qualidade.

\section{AGRADECIMENTOS}

Ao Museu Botânico de Curitiba, na pessoa de Osmar dos Santos Ribas, pela identificação da espécie, e ao técnico agrícola com habilitação em florestas Ademir Schmitd, pela participação. 


\section{REFERÊNCIAS}

ALEXANDRE, R. S.; JÚNIOR, A. W.; NEGREIROS, J. R. S.; BRUCKNER, C. H. Estádio de maturação dos frutos e substratos na germinação de sementes e desenvolvimento inicial de plântulas de jabuticabeira. Revista Brasileira de Agrociência, Pelotas, v. 12, n. 2, p. 227-230. 2006.

ANDERSEN, O.; ANDERSEN, V. U. As fruteiras silvestres brasileiras. Rio de Janeiro: Globo, 1988. 203 p.

ANDRADE, R. A.; MARTINS, A. B. G. Influence of the temperature in germination of seeds of jaboticaba tree. Revista Brasileira de Fruticultura, v. 25, n. 01, p. 197-198, 2003.

ANJOS, A. M. G. Morfologia e fisiologia da germinação de sementes de araçá-boi Eugenia stipitata ssp. sororia McVauger - Myrtaceae), uma frutífera nativa da Amazônia Ocidental. 1998. 78 f. Dissertação (Mestrado em Biologia Tropical e Recursos Naturais) - Universidade do Amazonas, Manaus, 1998.

ANTUNES, L. E. C.; RAMOS, J. D.; CHALFUN, N. N. J. Cultivo da jabuticabeira. Lavras: UFLA, 1995. 14 p. (Boletim de Extensão, 44).

BIRCHLER, T.; ROSE, R. W.; ROYO, A.; PARDOS, M. La planta ideal: revision del concepto, parametros definitorios e implementaction practica. Investigacion Agraria, Sistemas y Recursos Forestales, Madrid, v. 7, n. 1/2, p. 109-121, 1998.

CARNEIRO, J. G. de A. Produção e qualidade de mudas florestais. Curitiba: UFPR/FUPEF; Campos: UNEF, 1995. $451 \mathrm{p}$.

CARVALHO, N. M.; NAKAGAWA, J. Sementes: ciência, tecnologia e produção. Campinas, Fundação Cargill, 1980. 326 p.

CHALFUN, N. N. J.; PIO, R. Aquisição e plantio de mudas frutíferas. Lavras: UFLA, 2002. 19 p. (Boletim Técnico, 113).

DANNER, M. A.; CITADIN, I.; FERNANDES JÚNIOR, A. A.; ASSMANN, A. P.; MAZARO, S. M.; SASSO, S. A. Z. Formação de mudas de jabuticabeira (Plinia sp.) em diferentes substratos e tamanho de recipientes. Revista Brasileira de Fruticultura, v. 29, p. 179-182, 2007.

DANNER, M. A.; CITADIN, I.; FERNANDES JÚNIOR, A. A.; ASSMAN, A. P.; MAZARO, S. M.; DONAZZOLO, J.; SASSO, S. A. Z. Enraizamento de jabuticabeira (Plinia trunciflora) por mergulhia aérea. Revista Brasileira de Fruticultura, Jaboticabal, v. 28, n. 3, p. 530-532, 2006.

DICKSON, A.; LEAF, A. L.; HOSNER. J. F. Quality appraisal of white spruce and white pine seedling stock in nurseries. Forest Chronicles, v. 36, p. 10-13, 1960.

DONADIO, L. C. Cuidados com a jabuticabeira. O Estado de São Paulo, São Paulo, 23 nov. 1983. Suplemento Agrícola, p. 16.

DURYEA, M. L. Nursery cultural practices: impacts on seedling quality. In: DURYEA, M. L.; LANDIS, T. D. Forest nursery manual: production of bareroot seedlings. Corvallis: Martinus Nijhoff, 1984. p. 143-164.

GOMES, J. M.; COUTO, L.; LEITE, H. G.; XAVIER, A.; GARCIA, S. L. R. Parâmetros morfológicos na avaliação da qualidade de mudas de Eucalyptus grandis. Revista Árvore, v. 26, n. 6, p. 655-664, 2002.

GOMES, P. Fruticultura brasileira. São Paulo: Nobel, 1987. 446 p.

JOHNSON, J. D.; CLINE, M. L. Seedling quality of southern pines. In: DURYEA, M. L.; DOUGHERTY, P. M. (eds.). Forest regeneration manual. Netherlands: Klumer Academic, 1991. p. 143-162.

JOLY, A. B. Botânica: introdução à taxonomia vegetal. São Paulo: Companhia Editora Nacional, 1993. 777 p.

KHAN, A. A. Preplant physiological seed conditioning. Horticultural Review, New York, v. 13, n. 1, p. 131-181, 1992. 
KÄMPF, A. N. Seleção de materiais para uso como substrato. In: KÄMPF, A. N.; FERMINO, M. H. Substratos para plantas: a base da produção vegetal em recipientes. Porto Alegre: Genesis, 2000. p. 139-146.

LEGRAND, C. D.; KLEIN, R. M. Flora Ilustrada Catarinense. Herbário Barbosa Rodriques, Mirtáceas In: P. R. Reitz (ed.). Itajaí. p. 731-876, 1978.

LORENZI, H.; BACHER, L.; LACERDA, M.; SARTORI, S. Frutas brasileiras e exóticas cultivadas. São Paulo: Instituto Plantarum de Estudos da Flora, 2006. 640 p.

MAGALHÃES, M. M.; BARROS, R. S.; FINGER, F. L. Changer in non-structural carbohydrates in developing fruit of Myrciaria jaboticaba. Scentia Horticulturae, Amsterdam, v. 66, p. 17-22, 1996.

MANICA, I. Frutas nativas, silvestres e exóticas 1: técnicas de produção e mercado: abiu, amora-preta, araçá, bacuri, biribá, carambola, cereja-do-rio-grande, jabuticaba. Porto Alegre: Cinco Continentes, 2000. $327 \mathrm{p}$.

MATTOS, J. L. R. Frutíferas nativas do Brasil: jabuticabeiras. São Paulo: Nobel, 1983. 92 p.

Frutos indígenas comestíveis do Rio Grande do Sul. Porto Alegre: Secretaria da Agricultura, 1978. 31 p. (Publicações \pm PRNR, 1).

MENDONÇA, R. M. N. Maturação, secagem e armazenamento de sementes e propagação vegetativa de jaboticabeiras (Myrciaria sp.). Viçosa, 2000. 136 p. Tese (Doutorado). Universidade Federal de Viçosa.

MEXAL, J. L.; LANDIS, T. D. Target seedling concepts: height and diameter. In: TARGET SEEDLING SYMPOSIUM, MEETING OF THE WESTERN FOREST NURSERY ASSOCIATIONS, GENERAL TECHNICAL REPORT RM-200, 1990, Roseburg. Proceedings... Fort. Collins: USDA, Forest Service, p. 17-35. 1990.

MIELKE, J. C.; FACHINELLO, J. C.; RASEIRA, A. Fruteiras nativas: características de 5 mirtáceas com potencial para exploração comercial. Hortisul, Pelotas, v. 1, n. 2, p. 32-36. 1990.

PARVIAINEN, J. V. Qualidade e avaliação de qualidade de mudas florestais. In: SEMINÁRIO DE SEMENTES E VIVEIROS FLORESTAIS, 1., 1981, Curitiba. Anais... Curitiba: FUPEF, p. 59-90. 1981.

PASQUAL, M.; CHALFUN, N. N. J.; RAMOS, J. D.; VALE, M. R. do; SILVA, C. R. de R. Fruticultura comercial: propagação de plantas frutíferas. Lavras: UFLA/FAEPE, 2001. 137 p.

POPINIGIS, F. Fisiologia de sementes. Brasília: AGIPLAN, 1977. 289 p.

RIBEIRO, J. E. L. S. Flora da Reserva Ducke: guia de identificação das plantas vasculares de uma floresta de terra firme na Amazônia Central. Manaus: INPA, 1999. 800 p.

ROSE, R.; CARLSON, W. C.; MORGAN, P. The target seedling concept. In: TARGET SEEDLING SYMPOSIUM; MEETING OF THE WESTERN FOREST NURSERY ASSOCIANTIONS, 1990, Oregon. Proceedings... Oregon: USDA, p. 1-9. 1990.

ROTMAN, A. D. Los Géneros Calycorectes, Hexachlamys, Myrciaria, Paramyrciaria, Plinia y Siphoneugena en la Flora Argentina. Darwiniana 24 (14): 157-185. 1982.

SCARPARE FILHO, J. A.; NETO, J. T.; COSTA, J. W. H.; KLUGE, R. A. Efeito do ácido indolbutírico no enraizamento de estacas herbáceas de jabuticabeira-sabará (Myrciaria jabuticaba), em condições de nebulização. Revista Brasileira de Fruticultura, Jaboticabal, v. 21, n. 2, p. 146-149, 1999.

SOBRAL, M. Alterações nomenclaturais em Plinia (Myrtaceae). Boletim do Museu Botânico de Curitiba, Curitiba, n. 63, p. 1-4, 1985. 